\author{
Submitted: \\ 24.05.2018 \\ Accepted: \\ 15.02.2019 \\ Published: \\ 28.06.2019

\section{Macroscopic and ultrasonographic anatomy of the rotator cuff layers} \\ Zbigniew Czyrny ${ }^{1}$, Bartłomiej Kordasiewicz², Maciej Kiciński², \\ Małgorzata Brzozowska ${ }^{3}$

\begin{abstract}
${ }^{1}$ Private Practice, High-Med., Warsaw, Poland
${ }^{2}$ Department of Trauma and Orthopedics, Professor Adam Gruca Independent Public Teaching Hospital in Otwock, Otwock, Centre of Postgraduate Medical Education, Warsaw, Poland

${ }^{3}$ Department of Forensic Medicine, Medical University of Warsaw, Warsaw, Poland Poland; e-mail: zbigniew.czymy@wp.pl
\end{abstract} \\ Correspondence: Zbigniew Czyrny, High-Med. ul. Kasprowicza 27 lok. 2, 01-817 Warsaw,
}

Keywords

rotator cuff, anatomy,

US

DOI: $10.15557 /$ JoU.2019.0017

\begin{abstract}
Aim: The aim of the study was to determine the thickness of the tendinous and capsuloligamentous layers in the supraspinatus and infraspinatus zones of the shoulder. Materials and methods: Anatomical and ultrasonographic assessment of three fresh anatomical specimens consisting of the humeral head together with the capsuloligamentous layer called the superior complex and the supraspinatus and infraspinatus myotendinous units cut off at the level of the scapular glenoid rim. The first specimen was dissected on the length of approx. $10 \mathrm{~mm}$ from the glenoid insertion - the superior complex insertional zone to the scapula. Distally anterior (coraco-humeral and gleno-humeral superior ligaments) and posterior (gleno-humeral superior posterior ligament) limbs are connected by a transversely oriented ligament called the rotator cuff cable. This structure, together with the rest of the superior complex, belongs to the capsuloligamentous layer of the rotator cuff. The two other specimens were dissected (superior complex from the myotendinous units) from the level of the glenoid rim to the humeral insertion. Then the three specimens were scanned by ultrasound in a water bath and the measurements of both distinct layers were taken. Results: The rotator cuff in the supraspinatus and infraspinatus zone is a two-layer structure. The thickness of the tendinous and the capsuloligamentous layer is comparable. Conclusions: It may be concluded that the rotator cuff in the supraspinatus and infraspinatus zone is a two-layer structure, with the outer myotendinous layer, and the inner capsuloligamentous layer, which is called the superior complex. Since the thickness of these layers is comparable, it is important to bear in mind that the superior complex is an important part of shoulder biomechanics. Two different structures are found here - tendinous (dynamic) and capsuloligamentous (passive).
\end{abstract}

\section{Aim of the study}

The aim of the study was to determine the thickness of the tendinous and capsuloligamentous layers in the supraspinatus and infraspinatus zone using ultrasound.

\section{Materials and methods}

The material consisted of three fresh humeral heads together with rotator cuff structures cut off at the level of the glenoid rim/labrum (Fig. 1, Fig. 2, Fig. 3). The specimens 
consisted of the humeral head together with rotator cuff structures - superior complex (which connects the glenoid rim with the greater tubercle of the humerus), as well as bellies and tendons of the supraspinatus and infraspinatus. Both layers were cut at the level of the glenoid rim. In the first specimen, the tendinous layer was separated from the superior complex (for the first time described by Pouliart et al. (1) approx. $10 \mathrm{~mm}$ laterally from the insertion to the glenoid, with the use of blunt tools (fingers and the blunt edge of a blade). This is the glenoid insertional zone of the superior complex. At this level, supraspinatus and infraspinatus muscle bellies were dissected from the superior complex. Distally, the anterior (coraco-humeral and superior glenohumeral ligaments) and posterior (gleno humeral superior posterior ligament) limbs are connected by a transversely oriented ligament called the rotator cuff cable. This structure as well as the rest of the superior complex belong to the internal capsuloligamentous layer of the cuff. In specimens 2 and 3, the superior complex was separated from the myotendinous units all the way to the humeral insertion, using sharp tools (blade, scissors). The easiest dissection is where the superior complex is fused with muscle bellies of the supraspinatus and infraspinatus muscles - it is enough to separate the muscle from the superior complex.

In the second stage of the study, the specimens were examined by means of ultrasound in a water bath (Fig. 4, Fig. 5, Fig 6). Since this type of examination is free of artifacts generated in the superficial tissues (skin, subcutaneous fat, the deltoid muscle), the images are very clear. The US revealed (Fig. 4, Fig. 5, Fig. 6) delamination of layers of comparable thickness (tendinous and capsuleligamentous). The images were recorded as videoclips in .avi format as well as still .jpg images in longitudinal and transverse

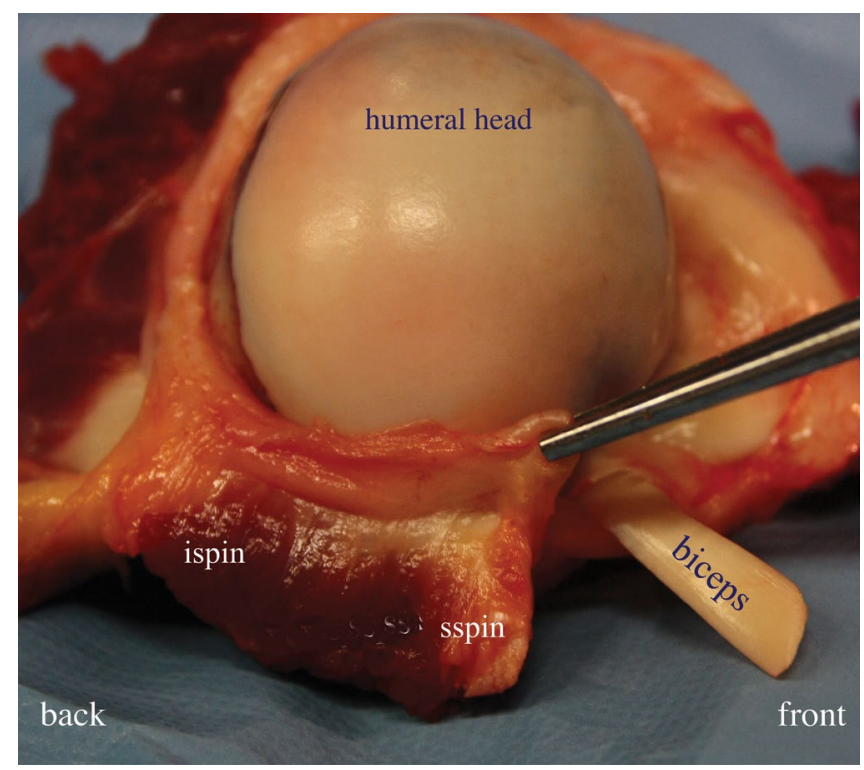

Fig. 1. Specimen 1. The rotator cuff and the humeral head. The tool is holding the anterior band of the superior complex. Sspinsupraspinatus zone; ispin - infraspinatus zone

planes - the latter were used for the measurements of the tendinous and superior complex layers.

\section{Results}

A total of 8 measurements of the thickness of tendinous and superior complex layers (four for each specimen) were performed in longitudinal and transverse planes of

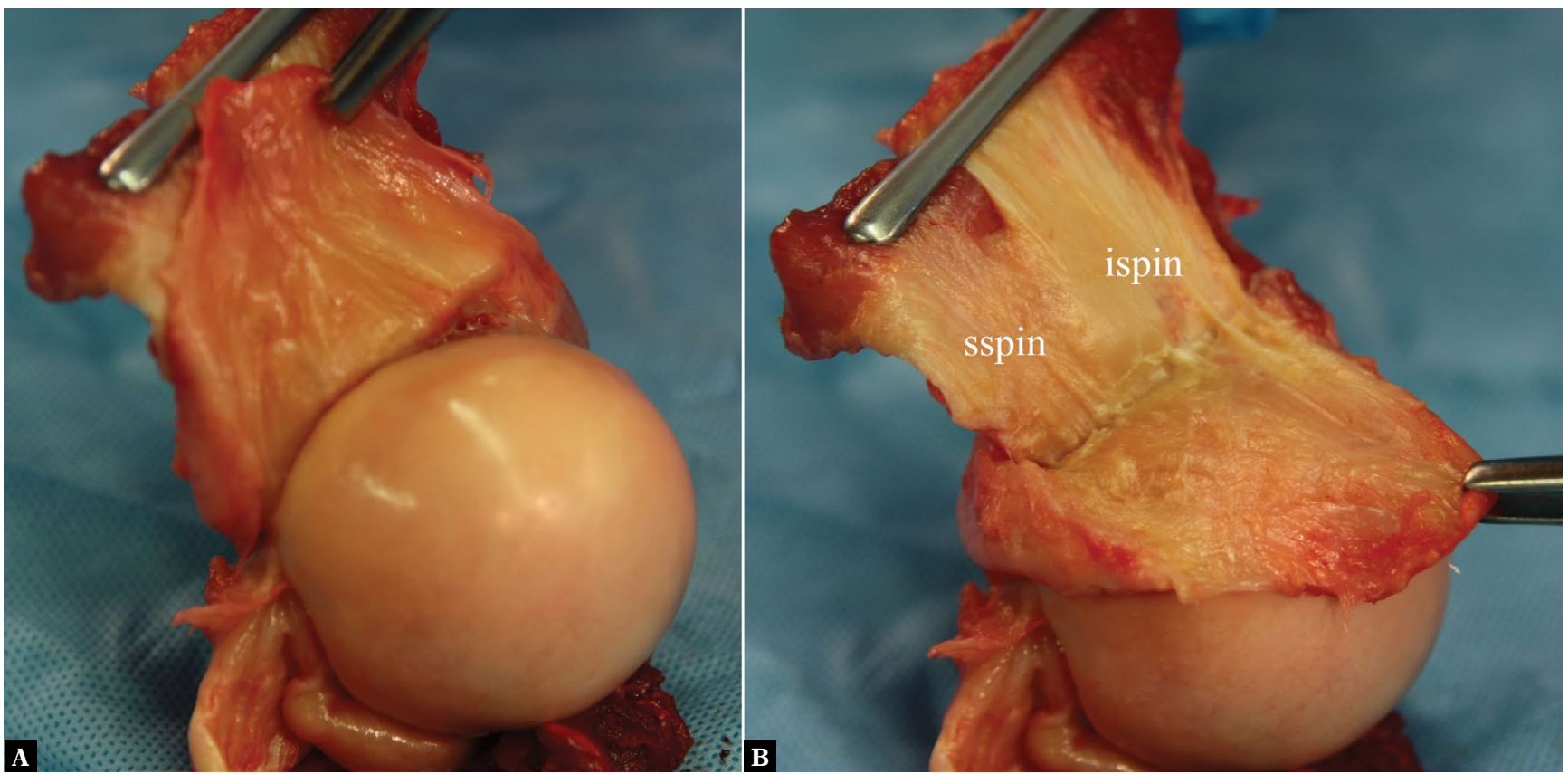

Fig. 2. Specimen 2 of the rotator cuff and the humeral head. A. Superior complex folded back from the humeral head; B. The superior complex covering part of the humeral head. After dissection of these layers, the tendons of the supraspinatus (sspin) and infraspinatus (ispin) appear on the inner surface of the muscles. Tool on $\mathbf{B}$ is holding the posterior limb of the superior complex 


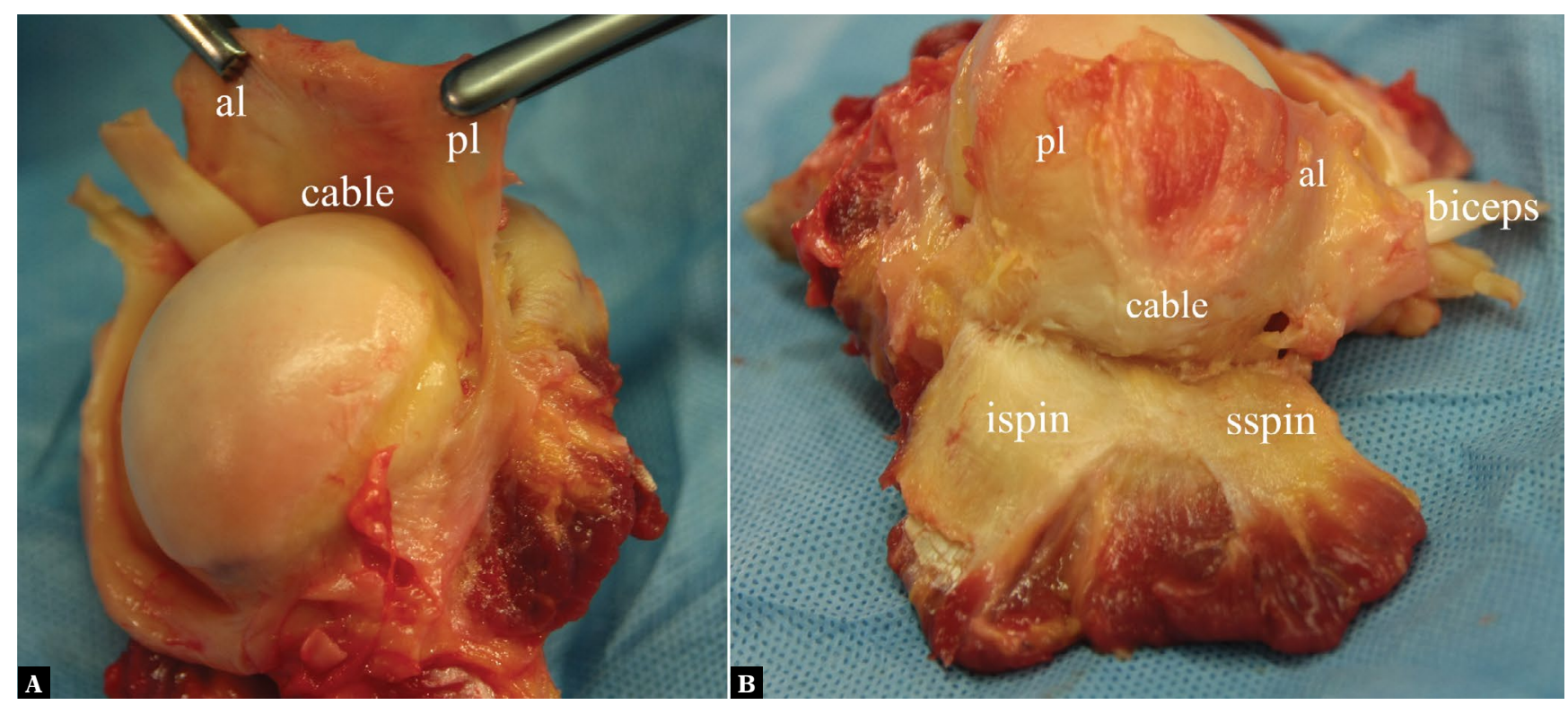

Fig. 3. Specimen 3 of the rotator cuff. A. A view seen by surgeons during humeral joint arthroscopy - the inner surface of the superior complex is seen. Al-anterior limb, $p l$ - posterior limb, cable - rotator cable (a ligament connecting distally anterior and posterior limb of the superior complex); B. The superior complex covering the humeral head, tendons of the supraspinatus (sspin) and infraspinatus (ispin) with muscle bellies separated from the superior complex

the supraspinatus and infraspinatus zones. Specimen 2: measurement $\mathrm{A}=4.2 \mathrm{~mm}$ and $3.8 \mathrm{~mm}$ in both planes the superior complex layer. Measurement $\mathrm{B}=3.5 \mathrm{~mm}$ and $2.9 \mathrm{~mm}$ in both planes - the tendinous layer. The superior complex layer was thicker than the tendinous one in both measurements. Specimen 3: measurement A $=1.9 \mathrm{~mm}$ and $3.2 \mathrm{~mm}$ in both planes - Superior complex layer. Measurement $\mathrm{B}=3.3 \mathrm{~mm}$ and $3.9 \mathrm{~mm}$ in both planes - the layer of the supraspinatus and infraspinatus tendons. Here, the tendinous layer was thicker than the superior complex.

\section{Discussion}

The description of the superior complex presented by Pouliart et al. encouraged me to undergo my own research on what is really seen in US and MRI when examining the rotator cuff.

The presence of a single-layer tendon and a layer of the superior complex is more or less precisely visible in US and MRI scans, which was already described in my previous report on rotator cuff diagnostics ${ }^{(2)}$. What we see is grossly the function of what we know of rotator cuff layers.

Both, the research presented by Pouliart and our study revealed a clear picture of the superior complex as an important functional structure underestimated by both surgeons and diagnosticians. What is more, the superior complex is still regarded as an internal layer of the supraspinatus and infraspinatus tendons.

The name of the superior complex comes from its complexity and location. It consists of two limbs - anterior and posterior. These bands are proximally inserted to the glenoid, and distally to the humerus. Distally, they are connected by a transverse ligamentous structure called the rotator cuff cable and then they insert to the greater tubercle. The anterior limb consists of the coracohumeral ligament and the superior glenohumeral ligament; the posterior limb consists of the superior posterior glenohumeral ligament, which was first described by Pouliart et al. The whole complex is additionally connected by capsular (multidirectional) fibers. The superior complex is responsible for the hanging of the humeral bone preventing it from luxation, but is also a resctrictor of the internal and external rotation of the humeral joint, which was described by Pouliart et al. ${ }^{(1)}$.

It seems that what was described many years ago by Pouliart et al. should be finally considered in the perception of the rotator cuff as a complex structure built of closely interconnected layers of tissues that are at the same time functionally different, and form layers of similar thickness.

Nimura et al. confirmed the comparable thickness of both rotator cuff layers ${ }^{(3)}$. The authors showed that the width of the humeral insertion of the tendinous (supraspinatus and infraspinatus) and capsuloligamentous (superior complex) layers differs depending on the location and measures approx. 1.2-11.4 $\mathrm{mm}$ for the tendinous layer and approx. 2.7-10.8 $\mathrm{mm}$ for the superior complex.

Both layers are well depicted in both US and MRI examinations $^{(4)}$, hence the need for popularization of this anatomical-diagnostic model of the rotator cuff. Therefore, updating of contemporary textbooks ${ }^{(5)}$ considering the "new" anatomy of the rotator cuff seems inevitable. 


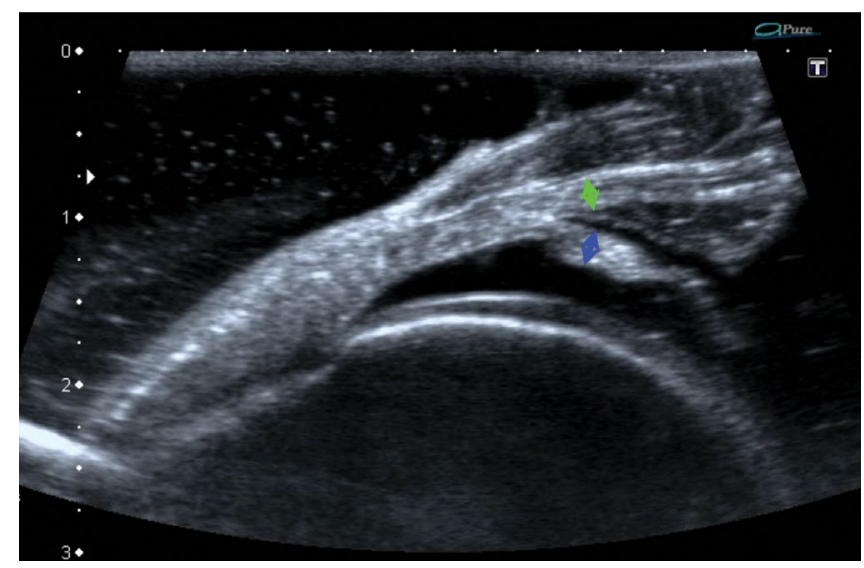

Fig. 4. US exam of the specimen 1. Longitudinal US section at the supraspinatus zone with separated tendinous (green arrows) and capsuloligamentous (blue arrows) layers. The depth of separation of the layers was approx $10 \mathrm{~mm}$. Both layers are similar in thickness. By permission of Czyrny $y^{(4)}$

\section{Conclusions}

What is currently called supraspinatus and infraspinatus tendon in the supraspinatus and infraspinatus zone is in fact approx. $1 / 2$ tendon and approx. $1 / 2$ superior complex (Fig. 7) ${ }^{(4)}$. Since there are obvious individual variations of the rotator cuff structures, the 50/50 division should not be treated stiffly.

Supraspinatus and infraspinatus tendons do not have layers - they are single-layered.

The outer layer of the rotator cuff is a tendinous one; the inner layer is a superior complex (Fig. 7).

Surgeons see the supraspinatus and infraspinatus tendons only by bursoscopy of the subacromial-subdeltoid bursa. Arthroscopy of the humeral joint may reveal the superior complex rather than the inner layer of the supraspinatus and infraspinatus tendons, as is currently thought.

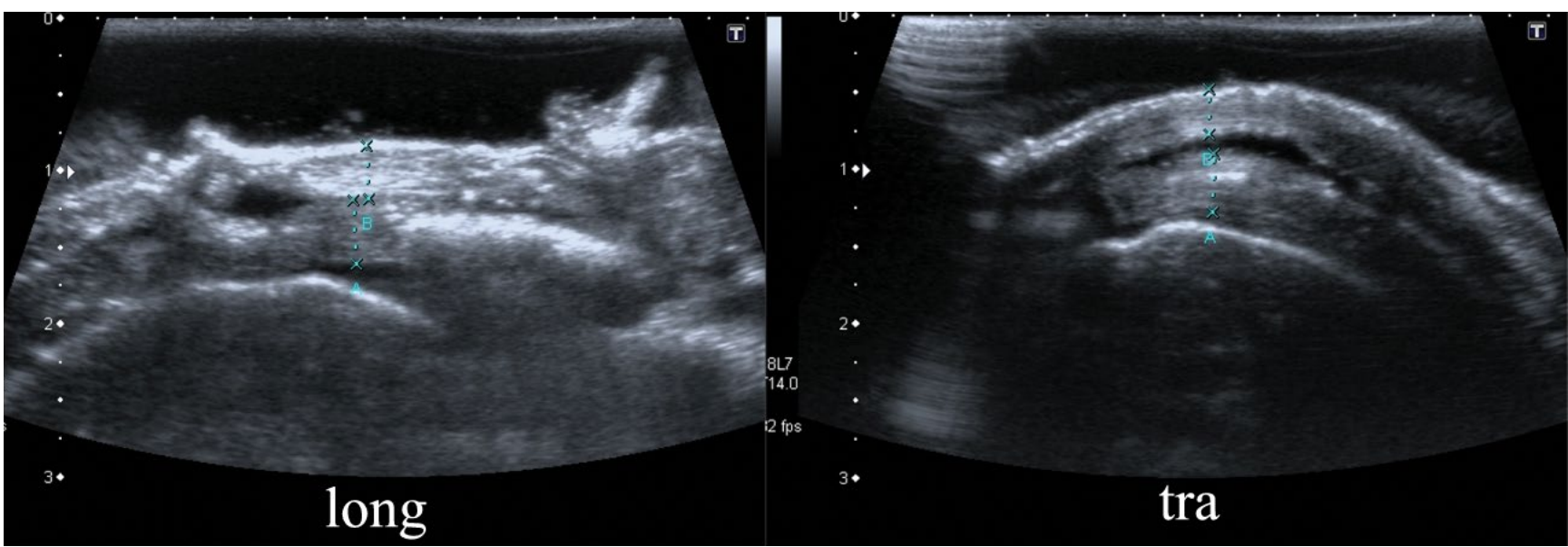

Fig. 5. Specimen 2. Longitudinal (long) and transverse (tra) sections of the supraspinatus/infraspinatus zone in a water bath. Measurement $A=4.2 \mathrm{~mm}$ and $3.8 \mathrm{~mm}$ in both planes - superior complex layer. Measurement B $=3.5 \mathrm{~mm}$ and $2.9 \mathrm{~mm}$ in both planes - tendinous layer. The superior complex layer is thicker than the tendinous one in both measurements

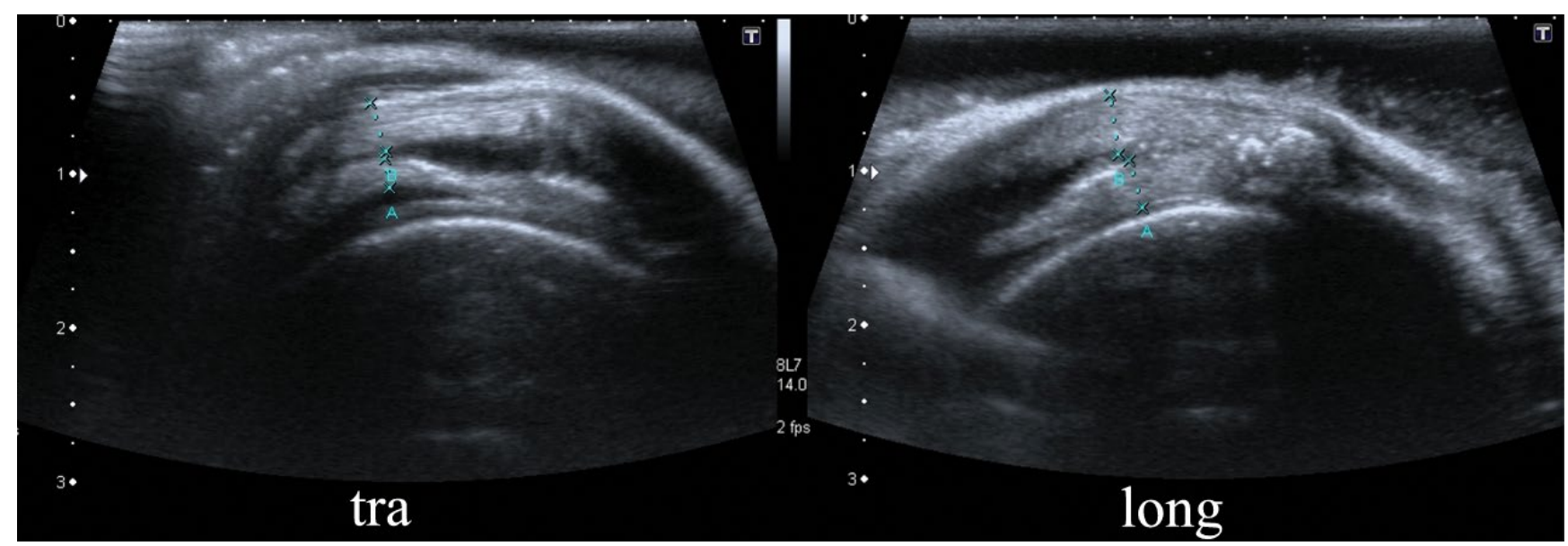

Fig. 6. Specimen 3. Longitudinal (long) and transverse (tra) sections in US examination in a waterbath. Measurement A = $1.9 \mathrm{~mm}$ and $3.2 \mathrm{~mm}$ in both planes - superior complex layer. Measurement $B=3.3 \mathrm{~mm}$ and $3.9 \mathrm{~mm}$ in both planes - layer of the supraspinatus and infraspinatus tendons. The tendinous layer is thicker than the superior complex in both measurements 


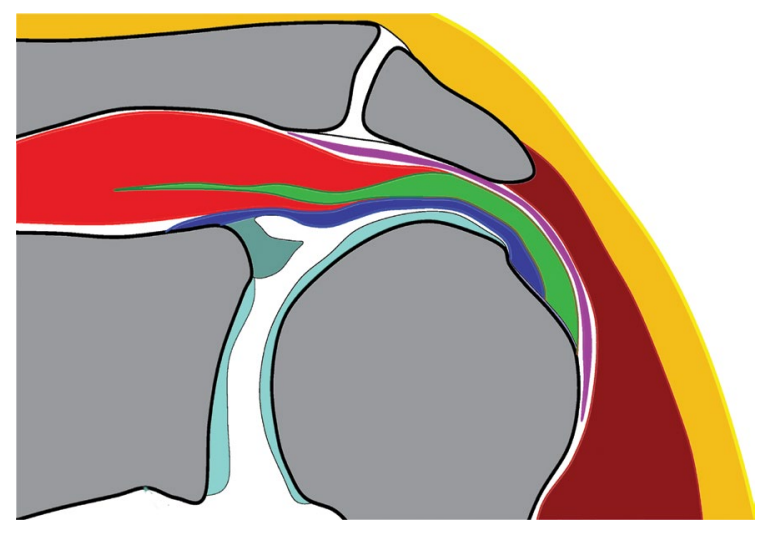

Fig. 7. A schematic representation of the rotator cuff structures. Longitudinal section of the shoulder in the supraspinatus or infraspinatus zone. Blue - superior complex, green - tendon, red - muscle belly, purple - subacromial-subdeltoid bursa. By permission of Czyrny(4)

\section{References}

1. Pouliart N, Somers K, Eid S, Gagey O: Variations in the superior capsuloligamentous complex and description of a new ligament. J Shoulder Elbow Surg 2007; 16: 821-836.

2. Czyrny Z: Diagnostic anatomy and diagnostics of enthesal pathologies of the rotator cuff. J Ultrason 2012; 12: 178-187.

3. Nimura A, Kato A, Yamaguchi K, Mochizuki T, Okawa A, Sugaya H et al.: The superior capsule of the shoulder joint complements
Therefore, it is important for both surgeons and diagnosticians to keep in mind that superficial tears are tendinous tears, and the tears of the deep layer of the rotator cuff are tears of the capsuloligamentous superior complex.

\section{Conflict of interest}

Authors do not report any financial or personal connections with other persons or organizations, which might negatively affect the contents of this publication and/or claim authorship rights to this publication.

the insertion of the rotator cuff. J Shoulder Elbow Surg 2012; 21 : $867-872$.

4. Czyrny Z: O systemach ścięgnistych mięśni. Kończyna górna. Spectrum Media, Warszawa 2014. ISBN 978-83-938977-0-4.

5. Bianchi S, Martinoli C: Ultrasound of the Musculoskeletal System. Springer, Berlin-Heidelberg-New York 2007. 